\title{
A Stochastic Optimization Approach to Optimal Bidding on Dutch Ancillary Services Markets
}

\author{
Laura Puglia \\ and Alberto Bemporad \\ IMT Alti Studi di Lucca \\ Lucca, Italy \\ Email: laura.puglia@imtlucca.it
}

\author{
Andrej Jokic \\ University of Zagreb \\ Zagreb, Croatia
}

\author{
Ana Virag \\ TuE Eindhoven \\ Eindhoven, the Nederlands
}

\begin{abstract}
The aim of this paper is to present a market design for trading capacity reserves (also called Ancillary Services, AS) and to introduce a strategy for the optimal bidding problem in such a scenario. In the deregulated market, the presence of several market participants or Balance Responsible Parties (BRPs) entitled for trading energy, together with the increasing integration of renewable sources and price-elastic loads, shift the focus on decentralized control and reliable forecast techniques. The main feature of the considered market design is its doublesided nature. In addition to portfolio-based supply bids and based on prediction of their stochastic production and load, BRPs are allowed to submit risk-limiting requests. Requesting capacity from the AS market corresponds to giving to the market an estimate of the possible deviation from the daily production schedule resulting from the day-ahead auction and from bilateral contracts, named E-Program. In this way each BRP is responsible for the balanced and safe operation of the electric grid. On the other hand, at each Program Time Unit (PTU) BRPs must also offer their available capacity under the form of bids. In this paper, a bidding strategy to the double-sided market is described, where the risk is minimized and all the constraints are fulfilled. The algorithms devised are tested in a simulation environment and compared to the current practice, where the double-sided auction is not contemplated. Results in terms of expected imbalances and reliability are presented.
\end{abstract}

\section{INTRODUCTION}

The profitable management of Balance Responsible Parties (BRPs), companies allowed to trade energy on the market, is based on a careful operation planning. Their portfolio usually consists of a set of generating plants, which can be fossil-fueled or powered with renewable sources, a set of internal loads and a group of (price-elastic) prosumers, small consumers and providers which modify their energy according to the electricity price.

A BRP has to allocate its output among different channels, and it can do that in several ways:

- long term bilateral contracts;

- the Day Ahead market, (also called Power Exchange or PX) that is the spot market for energy, whose result is

This work was partially supported by the European project E-PRICE: Pricebased Control of Electrical Power Systems, FP7-IST contract no. 249096. added to the long-term bilateral contracts forming the bulk daily schedule called E-Program;

- the Intra-Day market, the market for adjustments of the E-Program;

- the Ancillary Services (AS) market, the market for reserve capacity.

OTC contracts are nowadays highly exploited in the European markets [1]. The main motivation is that they bind sure incomes for BRPs and prevent power consumers with a high and steady request of power from being negatively exposed to the volatility of energy prices. However, the constantly growing focus for real-time optimization in power production brought the attention of researchers on the Day Ahead and on the Ancillary Services markets. The more sophisticated forecast and control techniques should be able to compensate for the volatility of renewable sources and prices, bringing a consistent advantage of the real time markets on the static long term contracts.

The task of submitting bids on the market is not trivial, as it involves a thorough and careful operation planning. The different markets are coupled, since if a BRP allocates a certain amount of power for the spot market, that amount is no longer available for bidding on the AS market.

Bidding on energy markets involves several sources of uncertainty, especially when a relevant part of provision is supplied by renewable sources, such as wind or solar cells. Other important stochastic variables affecting the bidding strategies are the high volatility of Day Ahead and Ancillary Services prices, and intermittent and price-elastic load. The presence of storage units could mitigate the uncertainty deriving from the use of renewable sources, but not always such systems are available.

In this paper we describe a novel market architecture designed for the AS bidding auction and we introduce a bidding strategy that can be used by BRPs to submit offers in this kind of market. The main characteristic of this market design is its double-sided nature, that gives to BRPs the possibility of placing, in addition to bids, also requests for capacity reserves, providing a confidence interval on the possible deviation 
from the contracted program. The main responsibility for the smooth operation of power systems is therefore shifted from the centralized TSO (or Transmission System Operator) to decentralized BRPs. This market structure has been first introduced in [2].

The goal of this paper is to describe a bidding strategy applicable to this framework and to present simulation results showing the validity of the new proposed solutions with respect to the standard baseline.

The paper is structured as follows. We will first give an overview of the state of the art in Section II. In Section III we will then report the proposed market architecture for reserve capacity. The description of a bidding strategy that can apply to this framework is given in Section IV. Simulation results showing the validity of this market structure with respect to the standard baseline are given in Section V, and conclusions are drawn in Section VI.

\section{STATE OF THE ART}

In the deregulated energy market, BRPs must submit profitmaximizing energy bids and offers for the spot market (PX) and for the regulating capacity or ancillary services market (AS). The market design highly influences the bidding strategies. The current Dutch market, which has been taken as the standard benchmark for our work, besides a set of bilateral contracts, consists of a Power Exchange, an Intraday market and an Ancillary Services market, as shown in Figure 1. For clarity, the Intraday market is neglected in this paper.

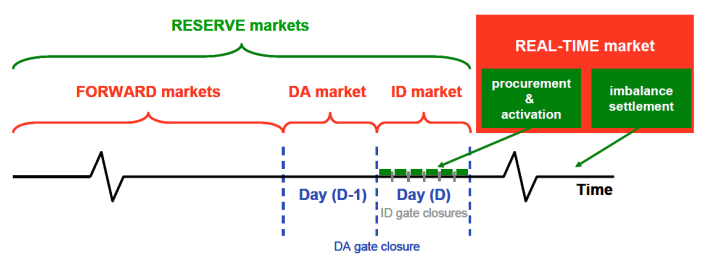

Fig. 1. The energy markets (source: [3])

One day before the delivery generators compose and submit bids to the Power Exchange (PX). Thereafter the market clearing price $(\mathrm{MCP})$ and the power volumes are assigned to each plant. Up to one hour prior to the delivery, BRPs submit offers for regulating capacity on the ancillary services market. Generators with installed power higher than $60 \mathrm{MW}$ are obliged to offer all the power they can increase or decrease by activating controllable generators. Those bids are sorted in ascending order as shown in Figure 2 and activated, the most convenient first, by the TSO to satisfy the real-time need for regulating capacity. BRPs supplying regulating capacity are rewarded at the marginal price, so the imbalance price is the price of the last activated bid. The imbalance price is the price of the last activated bid. BRPs incurring an imbalance pay their deviation from the E-Program at this price. An essential description of the background framework is contained in [4].

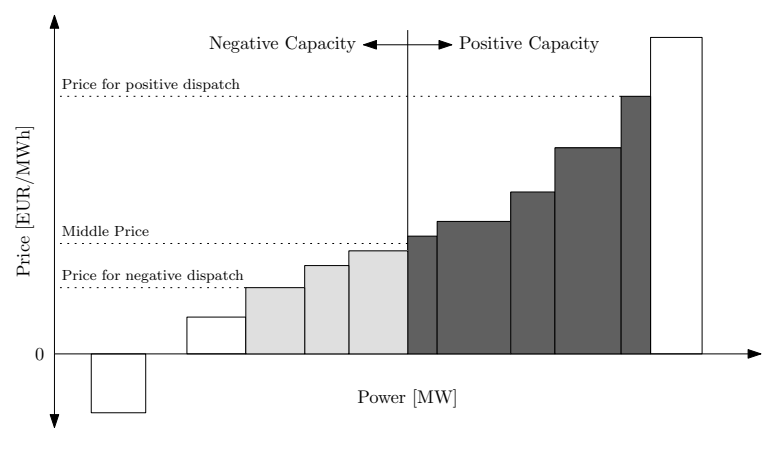

Fig. 2. The bidladder for ancillary services

Generally speaking, power plants that are deemed pricetakers tend to bid at the marginal cost. In fact, since the behavior of these plants is not supposed to influence the final market outcome, they just accept the cleared price as a result, under the condition that this price is higher of the marginal production cost, and therefore some profit is guaranteed. On the other hand, big power producers which are capable to influence the price for electricity have their private bidding strategies which cannot be revealed.

The bidding activity is crucial for the BRP's economic equilibrium, and involves the analysis of several sources of uncertainty. First, energy prices are highly volatile and can range from a few euros per MWh up to $1000 €$. Price forecasts occupy a central role, since a bad bidding strategy can lead to severe losses. Stochastic models of electricity prices, which are affected by high volatility and jumps, are presented in [5] and [6]. The latter considers the dynamical evolution of volatility and introduces parameter-varying models such as GARCH models. Second, the generator has its own load to satisfy, which is usually stochastic (see [7] for a possible approach to short-term load forecasts) as well as the available amount of renewable sources, over which only (more or less reliable) predictions can be given. Third, as the Day-ahead and the Ancillary Services markets mutually affect each other and the production is finite, a BRP has to decide where to allocate its capacity in order to optimize an economic objective which can be the pure expected profit or a riskbased signal. The coupling between Day-ahead and Ancillary Services markets represents one of the major limitations in this context. In many market arrangements, the plant has to set two independent bids at the same time, without knowing neither the Day-ahead price, nor the Ancillary services price, which on the other hand are strictly correlated. This may lead to global solutions that are far from the optimum, and the social welfare might be penalized. To overcome this limitations, we consider a market arrangement where Day-Ahead and Ancillary Services markets are executed in two subsequent sessions. A contribution to the market design considered in this work in which coupling between prices is avoided is contained in [8], where potential benefits and downsides of such a market structure are illustrated. The market design 
strategies proposed in the cited work include the execution of iterated spot and ancillary services auctions, thus implying multiple sequential bidding sessions to ensure convergence. A kind of decoupled bidding strategy is implemented on real systems in the Australian Energy Market [9]. Here, generators offers to the AS market are incremental price functions of the available reserve capacity, depending on the energy dispatched at the PX level. In the remainder, we can hence neglect the coupling between prices, since the outcome of the clearing process is known when the Ancillary Services bids are sent to the TSO.

\section{THE E-PRICE ELECTRICITY MARKET}

In this section we describe the market design which is the background of our work.

The day-ahead or Power Exchange (PX) auction in this work keeps the main features of the standard electricity market. BRPs submit their bids and offers and the $\mathrm{APX}^{1}$ operates the clearing of the market, whose output is an approximate schedule of the power production of the next day. From the day-ahead clearing process one can either result as a requestor or a supplier, and the corresponding income/outcome in one day is:

$$
I_{P X}=\sum_{h=1}^{24} E_{P X}(h) \lambda_{P X}(h)
$$

Where $\lambda_{P X}$ is always positive and $E_{P X}(h)$ is the energy delivered in hour $h$. Delivering/producing energy yields a profit and receiving/consuming costs, so a negative profit. To this value one must add the incomes from bilateral contracts and internal contracted loads, whose price is calculated on the basis of $\lambda_{E X}$. The main innovation is the double-sided concept for the ancillary services market, which is described in the next subsection.

\section{A. The Ancillary Services market}

In the framework envisioned in this work, Ancillary Services provision can be either passive (request $R$ ) or active (supply $S$ ). The request $R$ indicates all the energy a BRP wants to buy and can be bidirectional. It implies a negative cash flow, which means that the BRP is paying an amount of money. In particular:

- $R^{+}$implies positive request (BRP expects to be "long" and hence is willing to pay for additional absorption),

- $R^{-}$implies negative request (BRP expects to be "short" and hence is willing to pay for additional injection).

On the contrary, the supply $S$ indicates the surplus/deficit a BRP wants to sell, so that it wants to be paid for. It implies a positive cash flow, which means that the BRP is receiving an amount of money. In particular:

- $S^{+}$means positive supply (BRP is willing to be paid for producing more energy),

${ }^{1}$ APX-ENDEX is an European energy exchange, operating spot and futures markets for electricity and natural gas in the Netherlands, the United Kingdom and Belgium.

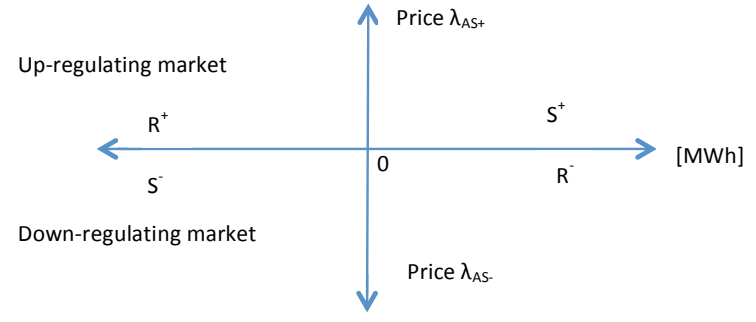

Fig. 3. Volumes and prices cleared at the AS market: upper part up-regulating, lower part down-regulating

- $S^{-}$means negative supply (BRP is willing to be paid for decreasing its production).

Bids for the AS market refer to Program Time Unit (PTU) of 15 minutes and can be sent up to one hour prior to the delivery. Let us define the following prices (in $€ / M W h$ ):

- $\lambda^{P X}$ is the Day-Ahead price,

- $\lambda^{A S+}$ is the price for injecting energy (upward direction),

- $\lambda^{A S-}$ is the price for sourcing energy (downward direction),

- $\lambda_{i m b}$ is the cost for causing an imbalance (upward or downward, not relevant at the moment).

Note that $\lambda^{A S+} \geq 0, \lambda^{A S-} \leq 0$ and always $\lambda_{i m b} \geq 0$. A BRP can participate in both the AS- and AS+ markets. When the AS markets are cleared, the prices $\lambda^{A S+}(k)$ and $\lambda^{A S-}(k)$ are determined, as well as the the net position of each BRP $E^{A S+}(k)$ and $E^{A S}(k)$. Therefore, from this process a BRP can either result as a supplier or a requestor. Payments for the only allocation of AS are proportional to the AS price $\lambda^{A S}$. Trading on the AS markets can therefore lead to a positive profit (S) or to a cost (R):

$I_{C A}=\sum_{k=1}^{N_{P T U}} a E^{A S+}(k) \lambda^{A S+}(k)+\sum_{k=1}^{N_{P T U}} a E^{A S-}(k) \lambda^{A S-}(k)$

Where $a$ is a design parameter of the market. In other words, the term $a$ might be seen as the cost for participating to the double-sided market. For example, if a BRP results as a requestor of positive energy for PTU $k$, its cleared capacity $E_{i}^{A S+}(k)$ is negative and it has to pay $a E_{i}^{A S+}(k) \lambda^{A S+}$ to reserve the quantity $E_{i}^{A S+}$ for regulating purposes. We use the notation for the signs of the cleared volumes and prices as shown in Figure 3.

In real time operations, the TSO sends in each $T_{P}$ seconds (in the simulation framework, $T_{P}=4$ ) a request signal called $\Delta P$ to BRPs, which is the request for varying the power output of controllable generators. If the need for upward regulating energy occurs, the TSO sends in positive $\Delta P$, on the contrary, if too much energy is present on the grid, the TSO transmits negative $\Delta P$. The signal $\Delta P$ is distributed among BRPs based on their cleared capacity. The profit obtained by the supply of regulating power on the AS in PTU $k$ is defined as follows: 
$I_{A S}(k)=\sum_{t=1}^{N}\left(w \Delta P(t) \lambda^{A S+}(t)+(1-w) \Delta P(t) \lambda^{A S-}(t)\right) \frac{T_{P}}{3600}$

where

$$
w= \begin{cases}1 & \text { if } \Delta P(t) \geq 0, \\ 0 & \text { if } \Delta P(t)<0\end{cases}
$$

where $N$ is the number of $T_{P}$ periods in a PTU. Note that $I_{A S}$ always denotes a profit, as the signs of $\Delta P$ and $\lambda^{A S}$ are always concordant.

\section{B. The imbalance system}

Any deviation from the scheduled E-Program is considered as imbalance (see [4]). Imbalances are settled by the TSO by means of a process called imbalance settlement.

For each PTU the imbalance price $\lambda_{i m b}$ is calculated, on the basis of $\lambda^{A S+}$ and $\lambda^{A S-}$ and of the control state. This parameter indicates the direction of the system imbalance and can take the values $+1,-1,0$ or 2 , as shown in Table I.

TABLE I

THE CONTROL STATE

\begin{tabular}{cc}
\hline$\Delta P$ & $\mathrm{CS}$ \\
\hline \hline always $<0$ & -1 \\
always $=0$ & 0 \\
always $>0$ & 1 \\
sometimes $<0$, sometimes $>0$ & 2 \\
\hline
\end{tabular}

A BRP is in imbalance when its control error $E_{C E}=$ $\int_{t=0}^{T} P_{r e f}(t)-P(t)$ deviates from 0 , where $P_{r e f}(t)$ is the power reference set-point deriving from the E-Program and AGC signal and $P(t)$ is the actual power output. Depending on $E_{C E}$ and on the cleared volumes of AS of BRP $i$, the imbalance costs/profits can be calculated. The imbalance price applied to positive deviations from the E-Program is calculated as $\lambda^{i m b,+}=\phi \lambda^{A S+}$, where $\phi=1+a+0.1$. The proportional factor must force the imbalance price to be higher than the AS price. Similarly, negative deviations from E-Program are penalized at the price $\lambda^{i m b,-}=\phi \lambda^{A S-}$. If a BRP has previously bought reserve capacity on the AS market, then as far as its deviation is within the cleared capacity the applied imbalance price is $\lambda^{A S+}$ or $\lambda^{A S-}$ (depending on the direction of the deviation). In case the imbalance exceeds the allocated capacity, the excess deviation is paid at $\lambda_{i m b}$.

In some market architectures passive balancing (i.e. deliberately causing imbalances which help the system) is rewarded, that is, even if the BRP is in imbalance it receives a payment if its deviation helps the system recover the normal conditions. In this work, passive balancing is neither rewarded nor penalized. Therefore, BRPs only aim at minimizing their own imbalances in real time. Nevertheless, passive balancing is applicable to our algorithms. In this case BRPs optimize in real time a given cost function and can intentionally go out of balance if that is profitable, that is, when the direction of their imbalance is opposite with respect to the system imbalance. A twoscale stochastic algorithm applying passive balancing has been devised, implemented and tested at BRP level in the E-Price framework and it is described in [10]. A possible approach to the design and implications of different passive balancing policies at system level are tackled in [11].

\section{THE DAY-AHEAD BIDDING STRATEGIES}

BRPs can be thought as aggregated companies producing and consuming energy and satisfying a certain amount of internal loads. The main goal of such companies is to maximize their own profit, while limiting the incurred risks. The concept of the project underlying this paper is to devise efficient algorithms, market architectures and ICT interfaces in order to shift the main responsibility for the reliability of the energy system from the centralized TSO to BRPs, and to economically incentivize them to guarantee the correct and safe operation on the grid. Each BRP aims at maximizing its own profit given by:

$\lambda_{B C} x_{B C}+\lambda_{D A} x_{D A}+\lambda_{A S} x_{A S}-\lambda_{i m b} x_{i m b}-\left(c_{2} p^{2}+c_{1} p+c_{0}\right)$

where:

- $\lambda_{B C} x_{B C}$ is the revenue from bilateral contracts,

- $\lambda_{D A} x_{D A}$ is the revenue from the day-ahead market,

- $\lambda_{A S} x_{A S}$ is the revenue from the trade on ancillary services markets,

- $\lambda_{i m b} x_{i m b}$ is the imbalance cost,

- the last term in brackets represents production costs, which is a quadratic function of power $p$.

By day-ahead strategies we mean the decision making process tackled by the BRP before delivering its produced energy. It includes the following tasks:

1) Bidding on the day-ahead market,

2) Scheduling a first indicative production profile by calculating the unit commitment schedule,

3) Bidding on the ancillary services market.

When submitting the day-ahead bid curve, BRPs must take into account the uncertainty deriving from AS prices (not yet disclosed), renewable production and loads. Since energy cannot be stored, the two markets are coupled, because what is sold at the day-ahead is no more available for trading on the AS market.

For this reason the bidding algorithm has been structured as a two-stage process, where in the first stage the day-ahead bidding curves are calculated, solving a scenario-based optimization problem for each hour of the following day, where a risk function based on (5) is minimized. More specifically, the algorithm aims at minimizing the Conditional Value at Risk (CVaR), a function used to indicate the expected losses trespassing a certain threshold (Value at Risk). Estimation of $\mathrm{CVaR}$ requires the definition of a loss function, which we have chosen as the negated profit. Hence in this case minimization of risk approaches to some extent the worst case minimization. 
The problem of deciding both the energy volume to offer on the market and the corresponding price is clearly bilinear ${ }^{2}$. In order to keep the problem linear, prices are fixed to some user-defined values and energy volumes are computed such that they are optimal for the chosen prices (a similar approach has been taken, e.g., in [12]). Let $\lambda_{1}^{P X}, \lambda_{2}^{P X}, \ldots, \lambda_{N}^{P X}$ be the sequence of fixed PX prices. For each $\lambda_{i}^{P X}, 2 L$ scenarios of possible prices for the Ancillary Services Market $\lambda_{i}^{A S}$, $i=1,2, \ldots, 2 L$ are generated, which constitute the second stage of the optimization problem. Then, the problem is solved for each of the generated prices $\lambda_{i}^{P X}$, obtaining the energy volumes $E_{i}^{P X}$ to offer on the market, with $i \in 1,2, \ldots, N$. Finally, the piecewise constant bid curve is constructed by interpolation as

$$
\lambda(E)=\left\{\begin{array}{rll}
\lambda_{1} & \text { if } \quad E_{1} \leq E<E_{2}, \\
\lambda_{2} & \text { if } \quad E_{2} \leq E<E_{3}, \\
\vdots & \vdots & \\
\lambda_{N-1} & \text { if } & E_{N-1} \leq E<E_{N}, \\
\lambda_{N} & \text { if } & E=E_{N} .
\end{array}\right.
$$

In order to solve the problem for the PX we assume a price taker point of view, that is, the considered BRP's offers do not influence the market significantly and the BRP accepts any couple (energy, price) decided by the market on the proposed bidding curve. Generating costs are approximated with an affine curve of the produced power. Stochastic scenarios of the optimization problem at this stage model the AS prices and their corresponding probability. Ancillary Services prices are generated based on historical data relating control energy prices (as differential to the Day-Ahead Market price) with the system imbalance, provided by Tenne $^{3}$. This relation, shown in Figure 4, is market driven and appears quite stable and robust over the years, hence being suitable to be used for modeling purposes. The left side of the graph is related to the case where the market is short, BRPs sell Energy to the TSO, and the ASM price is higher than the DAM price. Bids

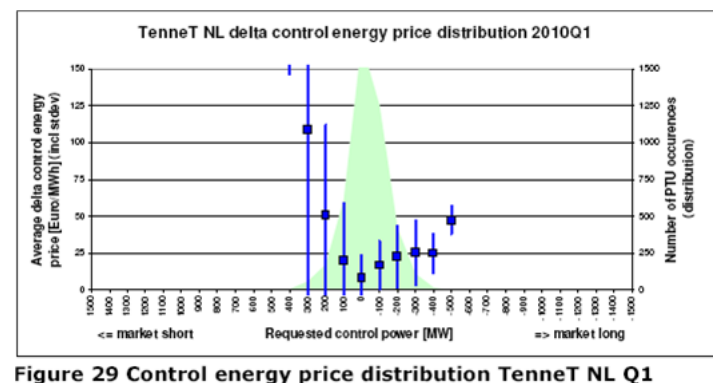

Fig. 4. Relation between day-ahead and ancillary services prices

are portfolio-based, that is, they are calculated based on the generating costs of the plants and their risk attitude. BRP risk

\footnotetext{
${ }^{2} \mathrm{~A}$ function is bilinear when it is linear in each of its variables. The simplest example is $f(x, y)=x y$.

${ }^{3}$ TenneT is the Dutch TSO, and also a partner in the E-Price project
}

attitudes are classified as small, medium or high, depending on the percentage of uncertainty a BRP wants to hedge against. After the clearing process, the E-Program is sent to each BRP. This is the basis for calculating the unit commitment algorithm, whose output is a 24 hour on/off schedule and average production profile for the following day.

Based on the E-Program and UC, up to one hour prior to delivery BRPs are free to submit offer and request curves for their residual capacity to the AS market. The same approach of the day-ahead bidding is used: a set of prices is fixed and optimal volumes to sell and buy are calculated for each of them, optimizing a stochastic function based on risk. Stochastic scenarios model the uncertain production that can be observed at the time of the delivery. Wind production and load are aggregated into one single "prosumption" stochastic variable (load is considered as negative stochastic production). Stochastic information on drift rate and standard deviation of the one-hour ahead error of wind and load are extracted from historical data. The approach used with respect to wind power is the so called persistence approach, meaning that the expected value of the wind production for the time of deployment is the one-hour ahead observation.

\section{EXPERIMENTAL RESULTS}

The aim of this section is to show the performance of the bidding techniques proposed in this paper in the singlesided and double-sided market architectures and with respect to the reference system. Data about AS realized prices, forecast and realized wind production and loads are provided by the Dutch TSO TenneT and by KEMA. Specifically, 4 data sets have been given, each differing in terms of wind forecast and size. Wind forecast can be perfect (in case the day-ahead forecast matches the realized wind production) or imperfect (the realistic situation accounting for some prediction error). The second difference stands in the size of the renewable production, which can be in line with the current standard or with the expected future production of 2030. The last aspect is considered to test the capability of BRPs for coping with the relevant wind increment expected in the future years.

The proposed day-ahead strategy has been tested in a simulation environment reproducing a national power system. The environment includes 7 BRPs with different production portfolios and risk attitudes, a TSO and a set of small power consumers/providers with elastic demand.

Plants consist of Combined Cycle Gas Turbines (CCGT) and wind farms. The simulated period is a time span of one day consisting of 96 PTUs with a length of 15 minutes.

The double-sided architecture has been compared to the actual single-sided structure for reserve markets, where no request can be submitted and imbalances are fully solved on the market based on a bid ladder (most convenient bids are activated first) as shown in Section II.

The effect of wind uncertainty and production has been tested by providing 4 data sets, each with different settings of wind forecast (perfect/imperfect) and production size (current/future). The parameter $a$ in (2) is set at $a=0.05$, 
consequently the imbalance penalty is fixed at $\phi=1.15$. In the simulated case studies the imperfect wind forecast condition relates to a situation where more wind than forecast is present in the system.

In each setting the double-sided architecture shows better performance with respect to profit and imbalance costs. By construction, AS prices are higher in the double-sided market and this encourages BRPs to allocate resources in a more efficient way. BRPs providing regulating capacity are rewarded more, thus increasing the overall benefit. To give an idea of the regulating capacity actually activated in real time, let us look at Figure 5, related to current wind production and imperfect forecast.

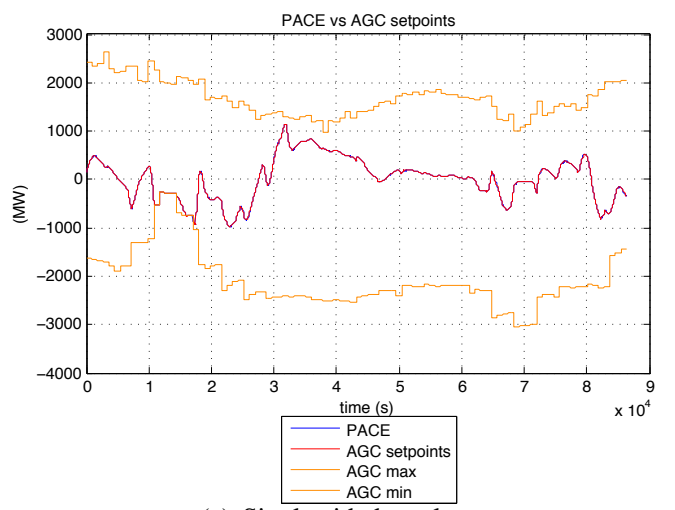

(a) Single-sided marlet

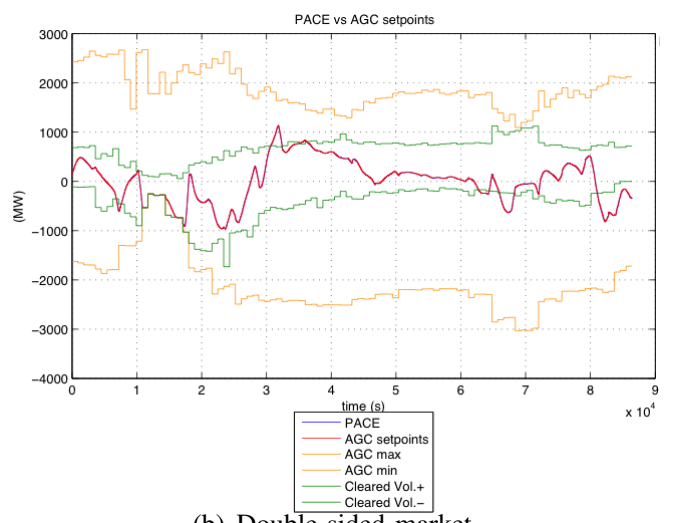

(b) Double-sided market

Fig. 5. AGC signal and allocated upward/downward capacity with current wind production and imperfect forecast

The red line is the aggregated signal (AGC signal) sent by the TSO to BRPs for regulating power. The trend of the signal is basically the same in the two settings, but in the doublesided case (Figure 5(b)) it is for most part included between the two green lines delimiting the cleared AS capacity. As far as the signal keeps between the two green lines, the system is prepared to react by delivering the previously allocated power. In case there is no sufficient cleared AS capacity, the TSO must resort to un-cleared bids. If not even this capacity suffices, the TSO must ask the help of neighboring countries. Also the price development is interesting in these two case studies, shown in Figure 6.

It can be noted in Figure 6(b) that double-sided prices are

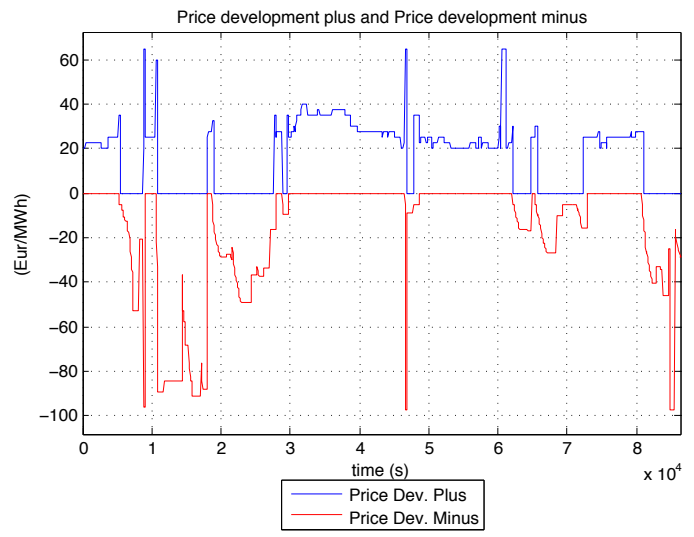

(a) Single-sided marlet

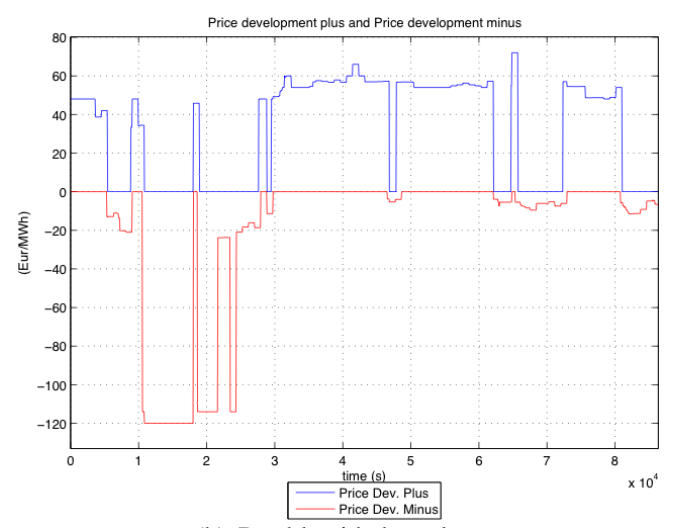

(b) Double-sided market

Fig. 6. ASPrice development with current wind production and imperfect forecast

constant over the PTU, they show a more regular trend and are in general higher with respect to the single-sided case (especially in the upward direction). This confirms that by construction, the double-sided market ensures more liquidity to the AS trade.

In perfect forecast conditions the AGC signal is as depicted in Figure 7. As expected, in the interval $\left[1 * 10^{4}, 3 * 10^{4}\right]$ seconds the AGC signal observed in Figure 7(a)-(b) is more balanced than in Figure 5, more specifically there is no need for the high downward capacity activated in imperfect forecast conditions. It can also be noted that in this situation, the cleared downward capacity is lower than the upward volume. This directly comes from the affine cost structure utilized in the optimization: due to the linearity of the cost function, decreasing the power output always leads to cost savings, as a consequence it is always preferable to balance any excess of power internally instead of resorting to the market. This means that downward request values tend to be small and in general limited to the cases when the risk of downward imbalance is threatening, which is more likely in imperfect forecast conditions. Price development in case of perfect forecast is reported in Figure 8. 


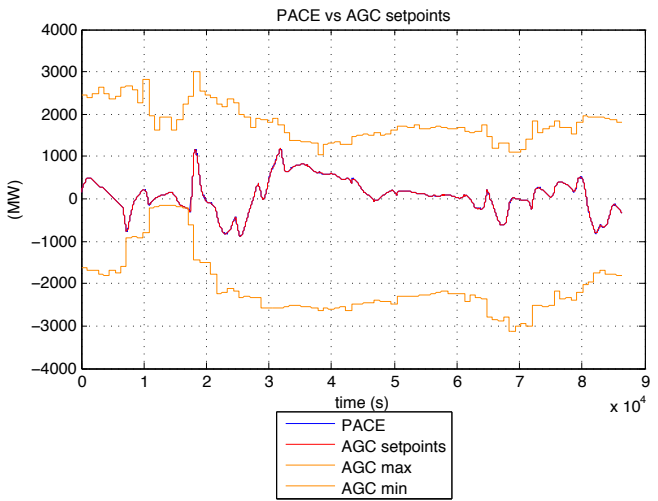

(a) Single-sided marlet

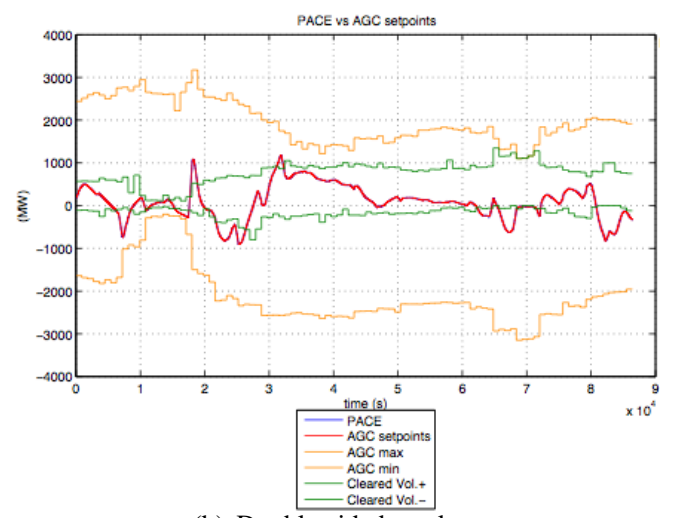

(b) Double-sided market

Fig. 7. AGC signal and allocated upward/downward capacity with current wind production and perfect forecast

\section{CONCLUSIONS}

In this paper, a market architecture for ancillary services provision has been presented and a bidding strategy that can apply to this market has been introduced. First, some overview on the state of the art and on the current benchmark we are aiming to improve on have been given. We have described the most important operations a BRP tackles from one the day up to one hour prior to the delivery, when the power production and allocation must be planned. These are delicate and risky tasks, because energy prices are extremely volatile and subject to fluctuations which are hard to absorb in the absence of energy storage devices in the grid. Moreover, the coupling between markets requires an even more attentive operation planning, since the energy sold on the Day-Ahead market cannot be used for other purposes.

The proposed solution is thoroughly designed to tackle the risk borne by BRPs and to support them in the decision making process. A scenario-based algorithm is devised where a linear stochastic problem is solved at every time unit (hour or PTU), to build the piecewise constant bid curve. A risk measure is minimized, in a way that reduces the risk of the BRP incurring great losses. Operating constraints such as minimum and maximum power setpoints, ramp rate limits and internal balancing are satisfied.

One of the most important features of the new market

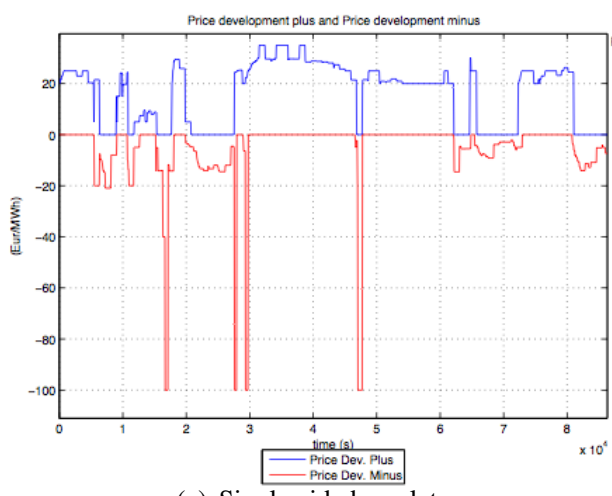

(a) Single-sided marlet

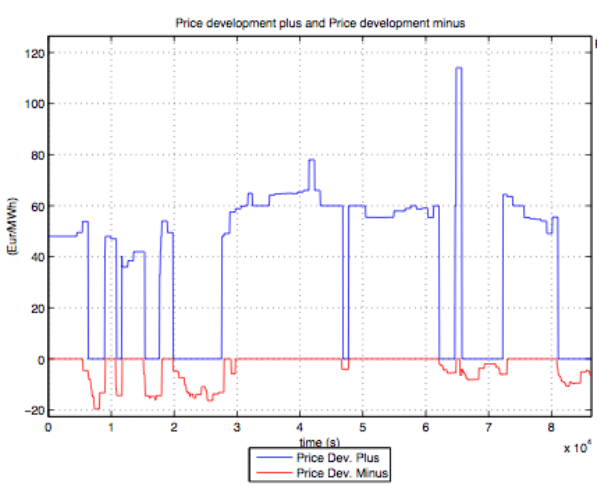

(b) Double-sided market

Fig. 8. ASPrice development with current wind production and perfect forecast

architecture is the introduction of AS requests quantities. These confidence intervals allow the system to be prepared to possible deviations in the prosumption by BRPs and react promptly.

\section{REFERENCES}

[1] K. Rademaekers, A. Slingenberg, and S. Morsy, "Review and analysis of eu wholesale energy markets," ECORYS Nederland BV, Report, 2008.

[2] P. Van den Bosch, "Reduced risk and improved economic operation of ancillary services," in 7th IEEE International Conference on the European Energy Market (EEM2010), Madrid, Spain, 23-25 June 2010.

[3] I. Lampropoulos, "Classification of viable control architectures," TU/e, Deliverable 1.1, 2010.

[4] TenneT-TSO, The Imbalance Pricing System as at 01-01-2001, revised per 26-10-2005, 2010.

[5] D. Most and D. Keles, "A survey of stochastic modelling approaches for liberalized electricity markets," European Journal of Operational Research, vol. 207, pp. 543-556, 2010.

[6] P. Theodorou and D. Karyampas, "Modeling the return and volatility of the greek electricity marginal system price," Energy Policy, pp. 26012609,2008

[7] S. Fan and R. J. Hyndman, "Short-term load forecasting based on a semi-parametric additive model," IEEE Transactions on Power Systems, vol. PP, no. 99, pp. 1-8, 2011.

[8] A. Virag, A. Jokic, R. Hermans, and P. Van den Bosch, "Combined bidding at power and ancillary service markets," in Energy Market (EEM), 2011 8th International Conference on the European , Zagreb, 2011, pp. 568-573.

[9] Guide to Ancillary Services in the National Electricity Market, 1st ed., Australian Energy Market Operator, 2010.

[10] P. Patrinos, D. Bernardini, A. Maffei, A. Jokic, and A. Bemporad, "Twotime-scale MPC for economically optimal real-time operation of balance responsible parties," in 8th IFAC Symposium on Power Plant and Power System Controls, Toulouse, France, 2012. 
[11] A. Jokic, P. van den Bosch, A. Virag, W. Hendrix, L. Puglia, W. de Boer, R. Vujanic, and F. Nobel, "Reliability and efficiency at global level in power systems," in 10th International Conference on European Energy Markets, 2013.

[12] S.-E. Fleten and T. K. Kristoffersen, "Stochastic programming for optimizing bidding strategies of a nordic hydropower producer," European Journal of Operational Research, vol. 181, pp. 916-928, 2007. 